\title{
Déterminants du dépistage volontaire, du partage du statut sérologique et prévalence de l'infection VIH chez les gestantes suivis à Parakou
}

\section{Determinants of voluntary screening, sharing of HIV status and prevalence of HIV infection in pregnant women followed in Parakou}

\author{
Alassani $\mathrm{A}^{1}$, Mama Cissé $\mathrm{I}^{1}$, Sidi Imorou $\mathrm{R}^{2}$, Gomina $\mathrm{M}^{1}$, Saké Alassan $\mathrm{K}^{1}$, Codjo $\mathrm{L}^{1}$, Dohou $\mathrm{H}^{1}$, Gandaho $\mathrm{P}^{1}$ \\ ${ }^{1}$-Département de Médecine et Spécialités Médicales, Faculté de Médecine, Université de Parakou, Bénin \\ 22-Département Mère-Enfant, Faculté de Médecine, Université de Parakou, Bénin
}

Auteur correspondant : ALASSANI Adébayo, Médecin interniste, Assistant Chef de Clinique, Faculté de Médecine, Université de Parakou, Mail: adebayoalassani@gmail.com

\begin{abstract}
Résumé
Objectifs : L'infection au VIH constitue un problème de santé publique. La transmission mère-enfant constitue l'un des principaux modes de contamination. La présente étude a pour objectif de décrire les obstacles au dépistage volontaire du VIH et au partage du statut sérologique avec le conjoint d'une part et de déterminer la prévalence de l'infection et d'identifier les facteurs associés d'autre part.

Patients et méthodes : Il s'agit d'une étude transversale à visée descriptive et analytique chez les gestantes suivies dans les maternités de Parakou. Les variables étudiées sont les déterminants du dépistage volontaire et du partage du statut sérologique, la prévalence du VIH. L'analyse des données a été faite par les logiciels Epi data 3.1 Epi-info 7 et Stata 11. Une p inférieure à 0,05 a été considérée comme significative.

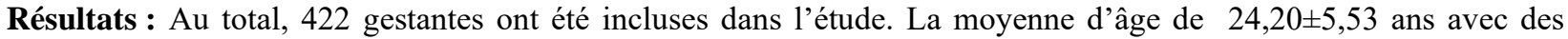
extrêmes de 17 ans et 40 ans. Parmi les 422 gestantes, 94,78\% ont accepté faire le dépistage volontaire du VIH afin de connaître son statut (49\%), se traiter (22,5\%) et protéger de l'enfant (17,5\%); 92,41\% ont accepté annoncé leur statut sérologique à leur conjoint pour bénéficier son soutien (42,6\%) et le fait qu'il soit leur confident $(29,2 \%)$. La prévalence de l'infection VIH est de 2,5\%. Aucun des facteurs étudiés n'est associé à l'infection.

Conclusion: La prévalence de l'infection VIH chez les gestantes suivies à Parakou est faible. Des obstacles entravent le dépistage volontaire et le partage du statut sérologique avec le conjoint.
\end{abstract}

Mots clés : Dépistage volontaire, Prévalence, Gestantes, Parakou

\begin{abstract}
Objectives: HIV infection is a public health problem. One of the main modes of infection is mother-to-child transmission. The purpose of this study is to describe the barriers to voluntary HIV testing and sharing of HIV status with a partner on the one hand, and to determine the prevalence of infection and identify associated factors on the other.

Design: This is a cross-sectional study with descriptive and analytical aims in pregnant women followed in maternities in Parakou. The variables studied are the determinants of voluntary screening and sharing of HIV status, HIV prevalence. The analysis of the data was done by the software Epi data 3.1 Epi-info 7 and Stata 11. A p less than 0.05 was considered to be significant.

Results: A total of 422 pregnant women were included in the study. The average age of $24.20 \pm 5.53$ years with extremes of 17 years and 40 years. Among the 422 pregnant women, $94.78 \%$ agreed to undergo voluntary HIV testing in order to know their status (49\%), treat themselves (22.5\%) and protect the child (17.5\%); $92.41 \%$ agreed to announce their HIV status to their spouse to receive their support (42.6\%) and the fact that they were their confidant (29.2\%). The prevalence of HIV infection is $2.5 \%$. None of the factors studied are associated with the infection.

Conclusions: The prevalence of HIV infection among pregnant women followed in Parakou is low. There are barriers to voluntary testing and sharing of HIV status with the spouse.

Keywords: Voluntary screening, Prevalence, Pregnant women, Parakou

L'infection à VIH demeure un problème mondial de santé publique spécialement en Afrique au sud du Sahara qui abrite $68 \%$ des 40 million de personnes vivant avec le VIH dans le monde [1]. Il s'agit d'un virus qui se transmet par plusieurs modes dont celui de la mère à l'enfant. Cette voie représente $9 \%$ des nouvelles infections au monde [2]. L'association du VIH à la

grossesse expose le foetus à une morbi-mortalité élevée liée au risque de contamination par ce virus dans la période de gravido-puerpéralité et peut compromettre l'évolution de la grossesse avec la fréquence des anémies chez la femme, la prématurité, le faible poids de naissance [3,4]. Au moins $20 \%$ des maladies dominantes chez les enfants de moins de 5 ans sont liées à la mauvaise santé et à la mauvaise nutrition de la mère, ainsi qu'à la qualité insuffisante des soins pendant la
\end{abstract}

\section{Introduction}


grossesse, l'accouchement et les premiers jours de la vie [5]. Le risque de transmission de l'infection de la mère à l'enfant varie entre 20 à $50 \%$ sans intervention que ce soit pendant la grossesse, le travail d'accouchement, la délivrance et l'allaitement maternel [6,7]. Avec une intervention appropriée et efficace, ce risque est réduit à moins de $2 \%$ dans la plupart des pays et s'est annulé dans les pays comme le Cuba [8]. Au Bénin, un programme de réduction de la transmission mère-enfant existe depuis 2012 dont la première phase est achevée en 2015 et une prolongation est faite jusqu'en fin 2020 [9]. Pour rendre efficace ce programme, il est important de dépister les femmes le plus tôt possible et ceci avant la grossesse dans la mesure du possible en vue d'un traitement précoce. Avant le dépistage, il est indispensable de faire un counseling afin d'amener la femme à réaliser le test. Plusieurs obstacles peuvent être observés au cours de cet entretien et peut compromettre la réalisation du test et aussi le partage du résultat avec le conjoint. La connaissance de ces différents obstacles et la mise en place des stratégies pour les surmonter seront des atouts à la réussite de ce programme. Peu de données existent au Bénin notamment dans le septentrion sur les déterminants du dépistage volontaire et le partage du statut sérologique. Il en est de même des données sur les facteurs associés à l'infection VIH à Parakou. La présente étude a été donc initiée afin de recenser les déterminants au dépistage volontaire et au partage du statut sérologique avec le conjoint et aussi de déterminer la prévalence et les facteurs associés à l'infection au VIH chez les gestantes.

\section{Patients et méthodes}

Cadre d'étude: l'étude s'est déroulée dans les maternités de Madina et de Kpébié sises dans la ville de Parakou.

Type et période d'étude: il s'est agi d'une étude transversale descriptive et analytique couvrant la période allant du 21 juin 2019 au 17 septembre 2019. L'étude analytique a seulement intéressé les facteurs associés à l'infection VIH chez les gestantes.

Population d'étude: elle est constituée des gestantes suivies dans les dites maternités pendant la période d'étude

Critères d'inclusion et de non inclusion: les gestantes ayant donné leur consentement à participer à l'étude ont été incluses. Celles incapables de répondre aux questions n'ont pas été incluses dans l'étude.

Echantillonnage : un recensement exhaustif des femmes a été fait.

Variables : elles ont regroupé 4 groupes de variables à savoir les déterminants du dépistage volontaire, les déterminants du partage du statut sérologique avec le conjoint quelque soit le résultat, la présence de l'infection VIH et les caractéristiques générales.
Les déterminants du dépistage volontaire : il s'est agi de recenser les raisons ayant motivé ou non l'acceptation du dépistage volontaire du VIH

Les déterminants du partage du statut sérologique avec le conjoint ont concerné les raisons qui peuvent inciter l'annonce de la sérologie VIH quelque soit le résultat à son partenaire ou l'inhiber en cas de résultat positif. Ils ont été recherchés chez toutes les gestantes y compris celles qui ont refusé le dépistage volontaire.

La présence de l'infection VIH a été faite par le test de diagnostic rapide VIKIA. En cas de résultat positif, une confirmation est faite par GEENIUS. Les gestantes ayant une sérologie VIH positive sont après l'annonce du résultat adressées au service de prise en charge de l'infection à VIH des dits centres pour un bilan préthérapeutique, une éducation thérapeutique et une prise en charge psychologique et médicamenteuse.

Les caractéristiques générales sont relatives aux données sociodémographiques, économiques, antécédents obstétricaux et à la connaissance sur le VIH.

Technique et outil de collecte de données : la collecte des données a été faite par l'entrevue face à face. Une fiche de collecte de données a été utilisée.

Traitement et analyse des données : les données ont été saisies à l'aide du logiciel Epi-Data version 3.1 après vérification de chaque fiche, elles ont été analysées à l'aide des logiciels Epi-info 7 et Stata 11. Une p value inférieur à 0,05 a été considérée comme significative. Seulement les caractéristiques générales associées à l'infection VIH ont été recherchées.

Aspects éthiques : la confidentialité des données est respectée lors de l'enquête. Après avoir expliqué aux femmes le but du travail, elles étaient libres de participer ou de ne pas participer à l'enquête et qu'une fois qu'elles ont accepté, elles étaient en droit de se désister après. L'anonymat est requis sur les fiches d'enquête. L'autorisation des responsables du centre est aussi obtenue. Le dépistage systématique de l'infection VIH fait partie du bilan prénatal.

\section{Résultats}

\section{Caractéristiques générales de la population d'étude}

La population d'étude est constituée de 422 gestantes ayant une moyenne d'âge de 24,20 $\pm 5,53$ ans avec des extrêmes de 17 ans et 40 ans. La majorité (82,9\%) avait des dépenses mensuelles du ménage inférieur au SMIG (Salaire Minimum Interprofessionnel Garanti). La quasitotalité $(99 \%)$ a entendu parler de l'infection au VIH; les sources d'information étaient les médias (40,7\%), le centre de santé $(36,8 \%)$ et les parents ou amis $(22,5 \%)$. S'agissant du niveau de connaissance, 24,2\% des gestantes avaient un niveau faible de connaissance générale sur le VIH, 32,7\% un niveau faible de connaissance sur la transmission mère-enfant du VIH et $25,1 \%$ un niveau faible sur la connaissance globale sur le $\mathrm{VIH}$. 
Tableau I: Caractéristiques générales de la population d'étude ( $\mathrm{n}=422$ )

\begin{tabular}{lcc}
\hline Caractéristiques générales & n & \% \\
\hline Age $<25$ ans & 242 & 57,3 \\
Gestité <3 & 272 & 54,5 \\
Parité <3 & 240 & 56,9 \\
Nombre d'enfants vivants $>1$ & 248 & 58,8 \\
Nombre d'enfants décédés = & 318 & 75,4 \\
Dépenses mensuelles $<$ SMIG & 350 & 32,9 \\
Instruction gestante Non instruite & 126 & 29,9 \\
Instruction époux= Non instruit & 94 & 22,3 \\
Situation matrimoniale=Mariée & 272 & 80 \\
Nombre de CPN antérieure = 0 & 36 & 8,5 \\
Informations sur le VIH = Oui & 418 & 99 \\
Connaissance générale du VIH =Elevée & 142 & 33,6 \\
Connaissance sur la transmission mère- & 210 & 79,8 \\
enfan = Elevée & & \\
Connaissance globale du VIH = Elevée & 170 & 40,3 \\
\hline
\end{tabular}

Déterminants du dépistage volontaire du VIH

Parmi les 422 gestantes, 400 ont accepté faire le dépistage volontaire de l'infection soit une proportion de $94,78 \%$. Les raisons ayant motivé cette action sont dominées par: connaître son statut (49\%), se traiter $(22,5 \%)$ et protéger de l'enfant $(17,5 \%)$. Les femmes qui ont refusé le dépistage volontaire sont au nombre de 22 soit une proportion de $5,22 \%$. Les raisons de ce refus sont principalement l'éviction des soucis $(45,4 \%)$ et l'absence des signes de l'infection VIH (27,3\%).

Tableau II : Déterminants du dépistage volontaire de l'infection VIH chez les gestantes $(\mathrm{n}=422)$

\begin{tabular}{|c|c|c|c|}
\hline Déterminants & & $\mathbf{N}$ & $\%$ \\
\hline \multirow{9}{*}{$\begin{array}{l}\text { Raisons de } \\
\text { l'acceptation } \\
\text { du dépistage } \\
\text { volontaire de } \\
\text { l'infection } \\
\text { VIH } \\
(\mathbf{N}=400)\end{array}$} & $\begin{array}{l}\text { Recommandations } \\
\text { de l'agent de santé }\end{array}$ & 14 & 3,5 \\
\hline & $\begin{array}{l}\text { Informer son } \\
\text { époux }\end{array}$ & 2 & 0,5 \\
\hline & $\begin{array}{l}\text { Informer déjà de } \\
\text { son statut }\end{array}$ & 2 & 0,5 \\
\hline & Aucune crainte & 2 & 0,5 \\
\hline & Maladie grave & 8 & 2,0 \\
\hline & $\begin{array}{l}\text { Connaître son } \\
\text { statut }\end{array}$ & 196 & 49 \\
\hline & Plus de sécurité & 16 & 4,0 \\
\hline & Pour se traiter & 90 & 22,5 \\
\hline & $\begin{array}{l}\text { Pour protéger } \\
\text { l'enfant }\end{array}$ & 70 & 17,5 \\
\hline Raisons du & Test déjà réalisé & 2 & 9,1 \\
\hline refus du & Absence de signes & 6 & 27,3 \\
\hline dépistage & Eviter les soucis & 10 & 45,4 \\
\hline $\begin{array}{l}\text { volontaire de } \\
\text { l'infection } \\
\text { VIH }(\mathbf{N}=22)\end{array}$ & $\begin{array}{l}\text { Maladie sans } \\
\text { traitement }\end{array}$ & 4 & 18,2 \\
\hline
\end{tabular}

\section{Déterminants du partage du statut sérologique avec son conjoint}

Parmi les 422 gestantes, 390 ont accepté annoncé leur statut sérologique à leur conjoint soit une proportion de $92,41 \%$. Les raisons ayant motivé cette action sont dominées la recherche du soutien de son conjoint $(42,6 \%)$ et le fait que son conjoint est son confident $(29,2 \%)$. Les femmes qui ont refusé le partage de leur statut avec leur conjoint en cas de sérologie VIH positive sont au nombre de 32 soit une proportion de 7,59\%. La principale raison de ce refus est la crainte de répudiation par son conjoint $(87,5 \%)$. (Tableau III)

Tableau III : Déterminants du partage du statut sérologique VIH avec son conjoint chez les gestantes $(\mathrm{n}=422)$

\begin{tabular}{llll}
\hline $\begin{array}{l}\text { Partage du statut sérologique VIH avec } \\
\text { son conjoint }\end{array}$ & N & \multicolumn{2}{l}{$\%$} \\
\hline Raisons de & Santé de l'enfant & 2 & 7,5 \\
l'acceptation du & Témoigner ma fidélité & 28 & 7,8 \\
partage du statut & Confident & 114 & 29,2 \\
sérologique VIH & Mauvaise maladie & 6 & 1,5 \\
avec son conjoint & Pour qu'il se protège & 36 & 9,2 \\
(N=390) & partenaires sexuelles & 14 & 3,9 \\
& Pour son soutien & 166 & 42,6 \\
& Pour qu'il se dépiste & 24 & 6,3 \\
\hline Raisons du & Il va me renvoyer & 28 & 87,5 \\
refus du & Il va me détester & 2 & 6,25 \\
partage du & Moquerie de ma & 2 & 6,25 \\
statut & coépouse & & \\
sérologique & & & \\
$\begin{array}{l}\text { VIH avec son } \\
\text { conjoint } \\
\text { (N=32) }\end{array}$ & & & \\
\hline
\end{tabular}

\section{Données relatives à l'infection $\mathrm{VIH}$}

Sur les 400 gestantes qui ont accepté faire le dépistage de l'infection VIH, 10 sont positive (VIH1) soit une prévalence de 2,5\% (Figure 1).

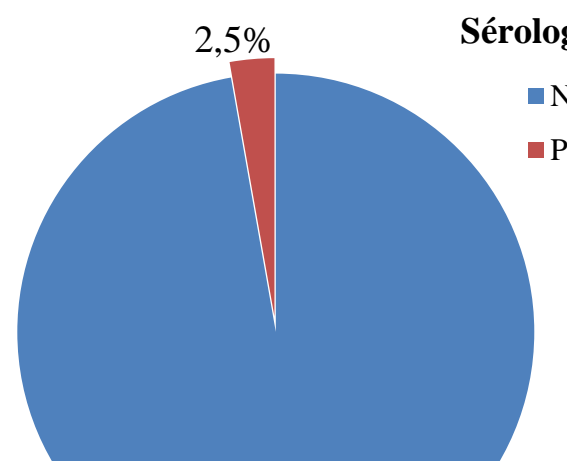

Figure 1: Prévalence de l'infection VIH chez les gestantes 
En considérant le statut positif ou non des gestantes qui ont refusé le dépistage, la prévalence de l'infection va varier entre 2,36 et $8 \%$. Parmi les 10 femmes, 7(70\%) ne connaissaient pas leur statut, $8(80 \%)$ sont dépistées au $2^{\text {ème }}$ trimestre de la grossesse et $2(20 \%)$ au troisième trimestre. Aucun facteur n'associé à l'infection VIH.

\section{Discussion}

L'infection au VIH constitue une pathologie tabou et terrifiante encore au Bénin avec la fréquence de fausses croyances. La présente étude a recensé les obstacles au dépistage volontaire et au partage du statut sérologie avec le conjoint d'une part et la détermination de la prévalence de l'infection VIH et les facteurs associés d'autre part chez les gestantes suivies dans deux principales maternités de la ville de Parakou.

La plupart $(94,78 \%)$ ont accepté faire le dépistage volontaire de l'infection VIH. Les raisons ayant motivé cette action sont dominées par: connaître son statut $(49 \%)$, se traiter $(22,5 \%)$ et protéger de l'enfant $(17,5 \%)$. Ce constat est similaire à celui rapporté par plusieurs auteurs. Dans l'étude de Diallo [10] à Kayes au Mali, 91\% des gestantes ont accepté le dépistage volontaire de l'infection VIH. La prévention de la transmission mère-enfant et l'importance du dépistage au cours de la grossesse ont été rapportées respectivement dans $90,1 \%$ et $86,6 \%$ par les gestantes à Abuja au Nigéria comme motifs de dépistage volontaire du VIH [11]. Les gestantes qui ont refusé le dépistage volontaire ont avancé comme raisons principales les soucis et l'absence de signes. Bibiana [11] dans son étude rapporte la stigmatisation et la discrimination $(87,3 \%)$ et le problème de confiance $(63,3 \%)$ comme principales raisons du refus de dépistage volontaire du VIH. Il est important de sensibiliser ces femmes afin qu'elles acceptent le dépistage volontaire tout en essayant de surmonter les obstacles rencontrés. Une fois le dépistage réalisé, les gestantes ont besoin d'un soutien aussi bien des agents de santé que des proches en cas de sérologie HIV positive. Parmi les proches, les conjoints ressemblent les personnes ressources; d'où la nécessité pour la femme de partager leur statut avec eux. Dans la présente étude, $92,41 \%$ des gestantes ont affirmé qu'elles informeront leur conjoint en cas de sérologie HIV positive. Dans l'étude de Diallo [10], 69\% des gestantes ont accepté informer leur conjoint de leur statut sérologique. La recherche du soutien de son conjoint $(42,6 \%)$ et le fait que son conjoint est son confident $(29,2 \%)$ sont les principales raisons avancées. Ceci montre l'importance du conjoint dans la prise en charge de l'infection VIH chez les gestantes. Cependant certaines gestantes ont affirmé ne pas informer leur conjoint principalement à cause de la crainte de répudiation $(87,5 \%)$. La crainte de divorce a été également rapportée par Diallo [10] chez les gestantes de la ville de Kayes au Mali.

La prévalence de l'infection VIH chez les gestantes dans la présente étude est $2,5 \%$. Cette prévalence est proche de celles de $1,72 \%, 2 \%$ et de $2,7 \%$ rapportées respectivement chez les gestantes à Bamako [12], au Bénin de 2006 à 2015 [9] et en Ethiopie [1]. Des prévalences plus faibles de cette infection ont été rapportées au Maroc (0,25\%) [13] et en Ethiopie (1\%) [14] chez les gestantes. Dans les pays, à forte endémicité VIH, la prévalence est plus élevée. C'est le cas de la prévalence de $8,3 \%$ rapportée à Jos au Nigéria [8] et 5,68\% à Abidjan en Côte d'Ivoire [4]. Toutes les gestantes sont infectées par le VIH1. La prédominance du VIH1 est aussi rapportée par Kouakou (95,9\%) [15] et Lonkeng (98\%) [16]. Seulement 30\% des gestantes infectées n'ignorent pas leur statut. Dans l'étude Ouassou [13] au Maroc, 46\% des gestantes infectées connaissaient leur statut. Les femmes sont dépistées pour la majorité $(80 \%)$ au $2^{\text {ème }}$ trimestre de la grossesse semblable aux résultats de Kouakou (60,3\%) [15] et de Lonkeng $(66,7 \%)$ [16]. Ceci implique le dépistage systématique de l'infection chez toutes les femmes en cas de désir de maternité. Aucun facteur n'est associé à l'infection à VIH chez les gestantes. Il s'agit donc d'une infection intéressant tout le monde quelque soit les caractéristiques sociodémographiques, économiques ou obstétricaux impliquant le dépistage systématique chez toutes les gestantes. Wirsiy [17] au Cameroun n'a retrouvé aucune association entre l'infection VIH et le niveau socio-économique. Dans l'étude de Omatola [18], l'âge, le statut matrimonial et la profession n'ont aucun lien avec l'infection VIH.

\section{Conclusion}

La prévalence de l'infection VIH chez les gestantes suivies à Parakou est faible. Des obstacles entravent le dépistage volontaire et le partage du statut sérologique avec le conjoint. Il est important de sensibiliser les gestantes en vue du dépistage systématique de l'infection et aussi du partage du statut sérologique avec le conjoint dans le but d'une prise en charge optimale afin de limiter les nouvelles infections.

Conflit d'intérêt : Aucun

Contribution des auteurs : AA a initié le travail, MCI a rédigé le manuscrit, SIR a effectué les analyses statistiques, GM, SAK, CL, DH et GP ont lu et corrigé le manuscrit

\section{Références}

1- Temesgen AB, Egata AD. Seroepidemiological patterns and predictors of hepatitis B, C and HIV viruses among pregnant women attending antenatal care clinic of Atat Hospital, Southern Ethiopia. SAGE Open Medicine 2020; 8: 1-9

2- Geremew D, Tajebe F, Ambachew S, Endalamaw A, Eshetie S. Seroprevalence of HIV among pregnant 
women in Ethiopia: a systematic review and meta-analysis

BMC Res Notes 2018; 11: 908-14

3- Akinbo FO, Olowookere TA, Oriakhi MO. Coinfection of Plasmodium falciparum and HIV among pregnant women in Edo State, Nigeria. J. Afr. Ass. Physiol. Sci 2019; 7 (1): 53-8

4- Effoh D, Koffi A, Adjobi R, Koffi S. Séroprévalence, prise en charge et pronostic foetal de l'infection à VIH au cours de grossesse au CHU de Cocody. Rev int sc méd-RISM 2018; 20(3): 223-8

5- Kasia JM, Noa NC, Belinga E, Kensoung H. Les Pathologies Maternelles en Grossesse au CHRACERH. Health Sci. Dis 2020; 21(4): 1-5

6- Tall BM. Evaluation du taux de transmission mère enfant du VIH chez les enfants nés de mères séropositives suivis au centre de santé de référence de la commune $\mathrm{V}$ du district de Bamako entre 2016 et 2017. Thèse de doctorat en médecine 2019; No 2154, 88 pages, Université des sciences des techniques et des technologies Bamako

7- Fouedjio JF, Fouelifack FY, Fouelifa LD, Mbu RE. Prevalence and associated factors of HIV infection among pregnant women attending antenatal care at the Yaoundé central hospital. Int J Reprod Contracept Obstet Gynecol. 2017 Jul;6(7):2698-703

8- Anyaka C, Oyebode T, Musa J, Isichei M, Anyaka I, Isichei C. HIV Prevalence amongst Pregnant Women Clients Attending Antenatal Clinic at the Faith Alive Foundation and PMTCT Centre, Jos Plateau State. World Journal of AIDS, 2016, 6, 59-64

9- Aguemon B, Sossa Jérôme C, Sopoh GE, Damien BG, Saizonou J, Houéto D et al.

Ten-Year-Trend of HIV Prevalence among Pregnant Women in Benin. Universal Journal of Public Health 2018; 6(2): 108-15

10- Diallo MC. Dépistage tardif du VIH en salle d'accouchement a l'hôpital Fousséyni Daou de Kayes : aspects clinique et épidémiologique. Thèse de doctorat en médecine 2019; No 2150, 89 pages, Université des sciences des techniques et des technologies Bamako.

11- Bibiana NE, Emmanuel PO, Amos D, Ramsey YM, Idris AN. Knowledge, attitude and factors affecting voluntary HIV counseling and testing services among women of reproductive age group in an Abuja Suburb community, Nigeria. Medical Journal of Zambia 2018; 45(1): 13-22

12- Diarra MS. Etude épidémiologique, Clinique et biologique de l'infection à VIH chez les gestantes suivies à la maternité du Centre de Santé de référence de la commune $\mathrm{V}$ du district de Bamako. Thèse de doctorat en médecine 2018; No 2003, 101 pages, Université des sciences des techniques et des technologies Bamako.

13- Ouassou S. Prise en charge de la femme enceinte séropositive au service de Gynécologie Obstétrique II au CHU HASSAN II (à propos de 24 cas). Thèse de doctorat en médecine 2018; No 2018, 175 pages, Université Sidi Mohamed Ben Abdellah

14- Ejeta E, Dabsu R. Prevalence of Hepatitis C Virus and HIV Infection Among Pregnant Women Attending Antenatal Care Clinic inWestern Ethiopia. Front. Med 2019; 5:366-73

15- Kouakou K, Doumbia Y, Djanhan L, Menin M, M'bro C, Djanhan Y. Séroprévalence, prise en charge et pronostic foetal de l'infection à VIH au cours de grossesse au CHU de Bouaké. Rev int sc méd -RISM2016;18,4:280-5.

16- Lonkeng AM. Séroprévalence du VIH chez les femmes enceintes au Centre de Santé de référence de la commune III du district de Bamako. Thèse de doctorat en médecine 2019; No 2103, 122 pages, Université des sciences des techniques et des technologies Bamako.

17- Wirsiy FS, Nsagha DS, Njajou O, Besong J. Prevalence and Associated Risk Factors of HIV among Pregnant Adolescents and Adolescent Mothers in the Kumbo West Health

District of Cameroon. Infect Dis Epidemiol 2019, 5(2): $1-11$

18- Omatola CA, Lawal C, Omosayin DO, Okolo MO, Adaji DM, Mofolorunsho CK and al. Seroprevalence of HBV, HCV, and HIV and Associated Risk Factors Among Apparently Healthy Pregnant Women in Anyigba, Nigeria. Viral immunology 2019; 32(4) : 186-191. 